\title{
shinyRGT: An R-Shiny application for extraction and visualization of Rat Gambling Task data
}

\author{
Andrew Li* \\ University of British Columbia
}

\author{
Georgios Karamanis ${ }^{\dagger}$ \\ Uppsala University
}

\author{
Tristan Hynes \\ ICORD
}

\author{
Catharine Winstanley \\ University of British Columbia
}

\begin{abstract}
The Rat Gambling Task (RGT) is a well validated rodent model of addiction-like behaviour. It is based on the Iowa Gambling Task (IGT) - a commonly used clinical assay to measure gambling-like behaviour. Rats choose between 4 options to earn as many sugar pellets as possible within $30 \mathrm{~min}$. Each option is associated with different reward sizes, but also the probability and duration of the time out punishment. The task is designed such that the optimal strategy for earning sugar pellets is to favour the low risk, low reward options. Consistently selecting the high risk, high reward options results in longer and more frequent time-out penalties. Currently, there is no specialized graphical user interface (GUI) designed to extract, clean, and process RGT data. The installation and use of existing tools are challenging for users lacking coding experience and can be extremely time consuming. To address these issues, we developed a free and open source R-Shiny application called shinyRGT, as a GUI for RGT data extraction, wrangling, and visualization. Clean and usable data can be easily extracted. As well, publication ready plots can be readily generated and annotated from user input. All generated tables can be downloaded as CSV files and generated graphs can be saved to local machines. shinyRGT is deployed at https://andrewcli.shinyapps.io/shinyRGT/for online use. The repository is available at https://github.com/andr3wli/ shinyapps.
\end{abstract}

\section{Statement of Need}

Addiction has become a public health epidemic, and costs the Canadian economy $\$ 46$ billion annually [9]. Drug addiction is also very hard to treat as majority of individuals relapse after achieving abstinence. It is therefore important to understand what factors render people vulnerable to addiction before we can explore potential treatments. Because the human condition is multifaceted and extremely complex, pre-clinical models of drug addiction have seen limited success [7]. Fortunately, in response, rodent models of drug addiction and new neural manipulation tools have made significant progress allowing for more precise examinations of the neurobiology underlying pro-addictive behaviours in animals $[5,6,12,18,24,25]$.

One such advances is the RGT, a model for pro-addictive decision making based on the IGT. It is clear that the RGT is a useful model because it has provided significant insight into drug addiction via probing different neurotransmitters including dopamine $[2,3,21,22,26,30]$, norepinephrine [2,22], serotonin [2,30], and the opiod system [10]. Given its popularity and usefulness, it is important to help streamline researchers' workflow. To date, the use of shiny apps in psychology and neuroscience are limited $[1,11,15,20]$. Specifically, there are no tools to help wrangle and visualize RGT data. The raw data files are quite messy and

\footnotetext{
*e-mail: andrewc.li@ubc.ca

†e-mail: georgios.karamanis@neuro.uu.se
}

requires multiple softwares to get clean and useable files. As well, researchers without a background in $\mathrm{R}$ will find it challenging to wrangle such messy data. Due to these limitations, there is a need for an alternative method to streamline the processing of RGT data.

Using the shiny framework [8], we present shinyRGT: a GUI software for interactive data wrangling and visualization of RGT data. shinyRGT was designed to facilitate the data cleaning, analysis, and visualization of RGT data. Users are encouraged via UX design principles to make use of the diverse widgets in shinyRGT to customize and annotate the resulting clean data set and the appearance of output plots.

\section{Method}

\subsection{Rat Gambling Task}

The RGT is a well validated rodent risky decision-making test modeled after the IGT $[4,30]$. Rodents have 30 minutes to sample between four response holes, each of which was associated with distinct magnitudes and probabilities of sucrose pellet rewards or time-out punishments. The optimal strategy is to favour options which deliver smaller per-trial gains but lower time-out punishments, thus leading to more frequent awards and less frequent and shorter time-out punishments. Continuously picking the safer options will yield the most reward delivery on each option. Responses made during the intermission were recorded as premature responses - a measure of impulsively. This resulted in the illumination of the house light and a $5 \mathrm{~s}$ time-out penalty after which a new trial could be initiated. If a response was not made into one of the 4 holes during the 10 s stimulus presentation, the trial would be registered as an omission, after which a new trial would begin.

The risk score was calculated by taking the sum of the optimal choice options chosen subtracted by the sum of risky choice option chosen. Rodents with a score above 0 are categorized as optimal and rodents below were categorized as risky.

\subsection{DREADDs}

Because of the complex relationship between addiction and decision making, controlled studies are impossible to conduct in humans, making animal models critical. A new transformative technology, Designer Receptors Exclusively Activated by Designer Drugs (DREADDs) empower researchers to address complex research questions. In the behavioural neuroscience field, DREADDs are routinely used for decision making and goal directed behaviour as it grants researchers the ability to monitor and probe in neural processes with high precision as animals complete complex behavioural assays [19]. As shown in $[13,14]$, when used in tandem, DREADDs and RGT allow researchers to target, identify, and manipulate specific brain regions underlying decision making and drug addiction.

\subsection{Shiny App Implementation}

shinyRGT is built completely in R. Currently, shinyRGT depends on the following R packages: shiny, tidyverse, lubridate, shinythemes, shinydashboards, shinyWidgets, shinyjs, and shinycssloaders. We primarily intend for shinyRGT to be used on the Shiny server 


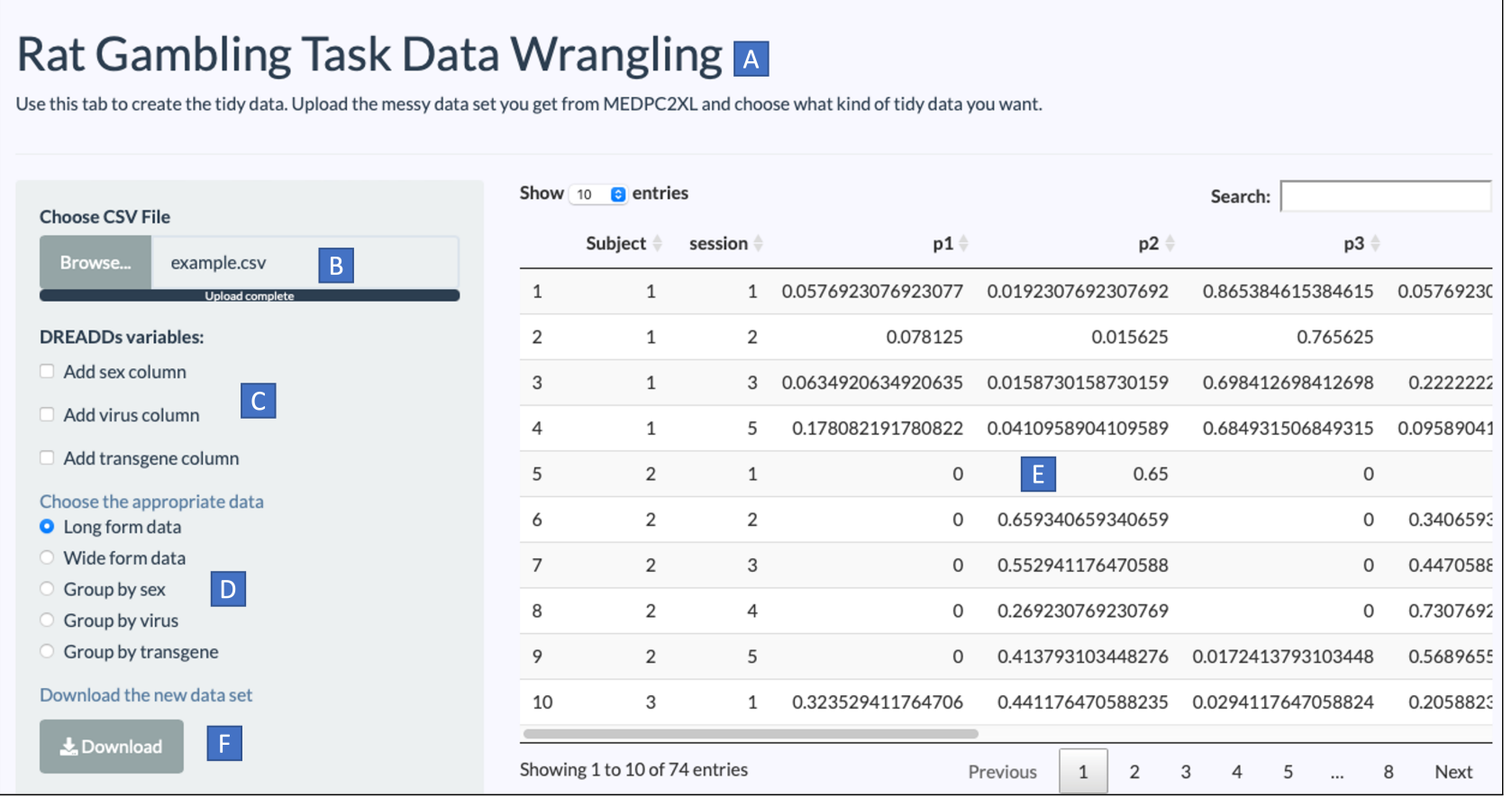

Figure 1: An overview of the data wrangling tab interface. (A) The title and a brief introduction the current tab. (B) A widget to allow users to upload messy data. (C) Additional variables that users can add. If selected, a new column will be created in the new data set. (D) Users can select the tidy data set that is appropriate for their analysis or visualization. (E) Users can view the resulting data set where they can filter and search the data. (F) Lastly, users can save the new data set as a CSV file.

via its URL [17] as it does not require $\mathrm{R}$ installation. However, it will launch locally from $\mathrm{R}$ provided the prerequisite packages are installed. The latest version can be cloned or forked from our GitHub page [16]. The latest version can be launched as follows:

\footnotetext{
shiny: :runGitHub (repo =

"andr3wli/shinyapps", subdir = "shinyRGT")
}

The shinyRGT application contains five main tabs located on the top left. The "Data Wrangling" and the "Data Visualization" tabs are the two main functionalities of shinyRGT and are responsible for producing the clean data and graphs. The "Data Wrangling" tab only accepts RGT data and while the "Data Visualization" tab was designed with the RGT in mind, it can be used to create plots from any data set. The "Help" tab provides instructions for the useage and installation of shinyRGT, including input data requirements and limitations. As well, it includes user manual guide. Lastly, the "Intro" tab provides a brief introduction to shinyRGT and a list of R packages and their dependencies.

\subsection{Data Wrangling}

Subject responses to each trial were recorded within the MED-PC software. Data were converted using MEDPC2XL into spreadsheet format for analysis. From, here users can import the RGT data into the data wrangling tab in shinyRGT. Once uploaded into the app, an analysis was conducted and applied within the app to calculate and create the relevant measures. These measures included: session number, max number of trials, sum of omissions, sum of premature responses, average time of collection latency, average time of choice selection, percent choice of the different holes selected (P1, P2, P3, $\mathrm{P} 4)$. These measures were ultimately used to calculate the risk score for each session for each subject. Additionally, if users used both sexes, a conditional check box allows users to indicate which subject were males. As well, if users used DREADDs, additional conditional check boxes allow users to indicate the virus and transgene status of the subjects. Theses conditional check boxes will create new columns in the new data set.

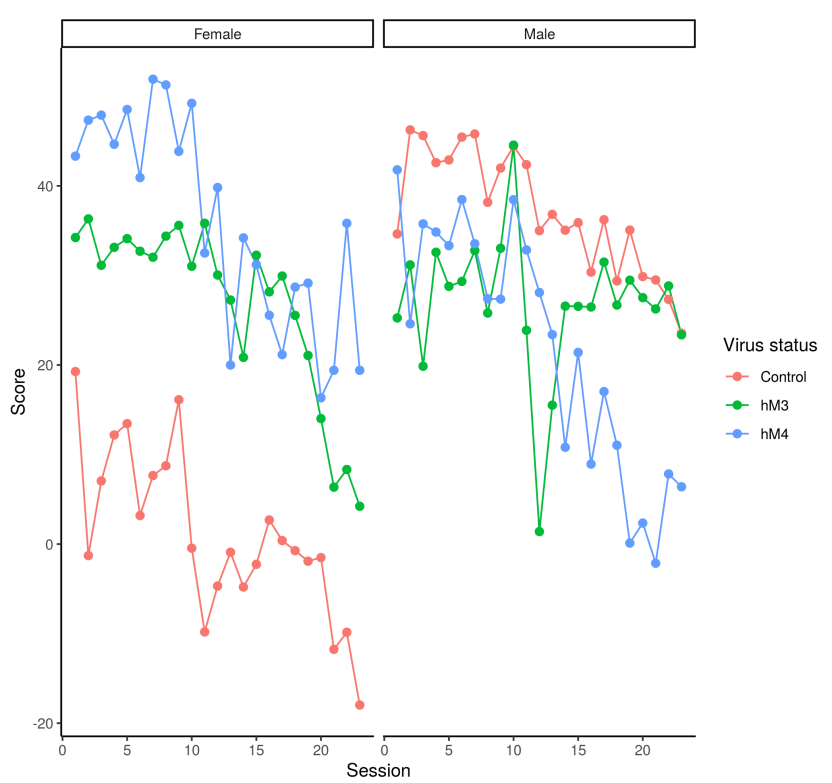

Figure 2: An example plot generated from RGT data via the data visualization tab. The $X$ axis shows the sessions and the $Y$ axis shows the risk score. The virus status is differentiated by color and sex is separated by panels. The title, $\mathrm{X}$ axis, $\mathrm{Y}$ axis, and legend title were annotated to be something human readable. 


\subsection{Data Visualizaton}

The data visualization tab follows the grammar of graphics principle $[27,29]$. Applied to visualization, grammar of graphics is used to describe and create a wide range of statistical graphics [29]. Using ggplot2 [28], the layered grammar of graphics approach is implemented in shinyRGT to produce publication ready plots specifically for RGT data. However, it can easily produce plots from other data sets as well. With established UX design principles in mind, we designed shinyRGT to follow the "Z" pattern of eye movement. Users start at the top left of the screen to the top right, then diagonally down to the bottom left to the bottom right. The use of the " $Z$ " pattern was ideal for our design because it has minimal text and graphic elements needed to organized by their priority [23]. The user inputs at the top left are crucial inputs to create the plot while inputs at the top right and the bottom are not crucial. At the top left, users are asked to provide a data source of the information to be visualized. Moving along the top, users are asked to provide the variables mapped to the $\mathrm{x}$ or $\mathrm{y}$ position and color aesthetics. After these essential elements, users have optional inputs to customize the plot. For example, users can annotate the title, subtitle, $\mathrm{x}$-axis, and the y-axis to something human readable. As well, users can choose to facet split the data into subsets, customize the theme, and plot a smooth line. Lastly, users can save the plot or the $\mathrm{R}$ code that produces the plot to their local machines.

\section{CONCLUSION}

shinyRGT is an R-shiny application for interactive creation of graphs using the grammar of graphics framework. We provide a free open source web application that provides a user-friendly graphical interface for users with limited programming experience. We have deployed shinyRGT at www.andrewcli.shinyapps.io/shinyRGT/, which enables non-R users to use shinyRGT without any installation. We encourage users to run shinyRGT on their personal computers. Advanced users can also contribute to the development of shinyRGT, the source code is avalible on GitHub.

\section{REFERENCES}

[1] S. Andrews, D. A. Ellis, H. Shaw, and L. Piwek. Beyond self-report: tools to compare estimated and real-world smartphone use. PloS one, 10(10):e0139004, 2015.

[2] P. J. Baarendse, C. A. Winstanley, and L. J. Vanderschuren. Simultaneous blockade of dopamine and noradrenaline reuptake promotes disadvantageous decision making in a rat gambling task. Psychopharmacology, 225(3):719-731, 2013.

[3] M. M. Barrus and C. A. Winstanley. Dopamine d3 receptors modulate the ability of win-paired cues to increase risky choice in a rat gambling task. Journal of Neuroscience, 36(3):785-794, 2016.

[4] A. Bechara, A. R. Damasio, H. Damasio, and S. W. Anderson. Insensitivity to future consequences following damage to human prefrontal cortex. Cognition, 50(1-3):7-15, 1994.

[5] A. Belin-Rauscent, M. Fouyssac, A. Bonci, and D. Belin. How preclinical models evolved to resemble the diagnostic criteria of drug addiction. Biological psychiatry, 79(1):39-46, 2016.

[6] J. P. Britt and A. Bonci. Optogenetic interrogations of the neural circuits underlying addiction. Current opinion in neurobiology, 23(4):539-545, 2013.

[7] B. Chan, K. Kondo, M. Freeman, C. Ayers, J. Montgomery, and D. Kansagara. Pharmacotherapy for cocaine use disorder-a systematic review and meta-analysis. Journal of general internal medicine, 34(12):2858-2873, 2019.

[8] W. Chang, J. Cheng, J. Allaire, C. Sievert, B. Schloerke, Y. Xie, J. Allen, J. McPherson, A. Dipert, and B. Borges. shiny: Web Application Framework for $R, 2021$. R package version 1.6.0.

[9] C. S. U. Costs. Canadian substance use costs and harms 2015-2017, 2020.
[10] P. Di Ciano and B. Le Foll. Evaluating the impact of naltrexone on the rat gambling task to test its predictive validity for gambling disorder. PLoS One, 11(5):e0155604, 2016.

[11] D. A. Ellis and K. J. Renouf. Predicting fear of crime: Personality outperforms prior victimisation. The Journal of Forensic Psychiatry \& Psychology, 29(3):403-418, 2018.

[12] S. M. Ferguson and J. F. Neumaier. Using dreadds to investigate addiction behaviors. Current opinion in behavioral sciences, 2:69-72, 2015.

[13] T. Hynes, K. Hrelja, B. Hathaway, C. Hounjet, C. Chernoff, S. Ebsary, G. Betts, B. Russell, L. Ma, S. Kaur, et al. Dopamine neurons gate the intersection of cocaine use, decision making, and impulsivity. bioRxiv, 2020.

[14] T. J. Hynes, B. A. Hathaway, K. M. Hrelja, S. A. Ebsary, C. S. Chernoff, L. Ma, B. Russell, S. Kaur, and C. A. Winstanley. Chemogenetic inhibition of ventral tegmental dopamine neurons prevents cocaineinduced deficits in decision making in both sexes. Pharmacological Reports, 71(6):1310, 2019.

[15] A. Li. shinyjackpot: Visualizing lottery gambling in a large canadian city. PsyArXiv, 2021.

[16] A. Li. shinyRGT GitHub repository. https://github.com/ andr3wli/shinyRGT, 2021. Online; accessed 25 March 2021.

[17] A. Li. shinyRGT Online version. https://andrewcli.shinyapps. io/shinyRGT/, 2021. Online; accessed 25 March 2021.

[18] S. V. Mahler, Z. D. Brodnik, B. M. Cox, W. C. Buchta, B. S. Bentzley, J. Quintanilla, Z. A. Cope, E. C. Lin, M. D. Riedy, M. D. Scofield, et al. Chemogenetic manipulations of ventral tegmental area dopamine neurons reveal multifaceted roles in cocaine abuse. Journal of Neuroscience, 39(3):503-518, 2019.

[19] B. L. Roth. Dreadds for neuroscientists. Neuron, 89(4):683-694, 2016.

[20] H. Shaw, D. A. Ellis, L.-R. Kendrick, F. Ziegler, and R. Wiseman. Predicting smartphone operating system from personality and individual differences. Cyberpsychology, Behavior, and Social Networking, 19(12):727-732, 2016.

[21] M. M. Silveira, E. Malcolm, M. Shoaib, and C. A. Winstanley. Scopolamine and amphetamine produce similar decision-making deficits on a rat gambling task via independent pathways. Behavioural brain research, 281:86-95, 2015.

[22] M. M. Silveira, W. S. Murch, L. Clark, and C. A. Winstanley. Chronic atomoxetine treatment during adolescence does not influence decisionmaking on a rodent gambling task, but does modulate amphetamine's effect on impulsive action in adulthood. Behavioural pharmacology, 27(4):350-363, 2016.

[23] M. Soegaard. Visual hierarchy: Organizing content to follow natural eye movement patterns, 2019.

[24] A. M. Stamatakis and G. D. Stuber. Optogenetic strategies to dissect the neural circuits that underlie reward and addiction. Cold Spring Harbor Perspectives in Medicine, 2(11):a011924, 2012.

[25] M. T. Stefanik, K. Moussawi, Y. M. Kupchik, K. C. Smith, R. L. Miller, M. L. Huff, K. Deisseroth, P. W. Kalivas, and R. T. LaLumiere. Optogenetic inhibition of cocaine seeking in rats. Addiction biology, 18(1):50-53, 2013.

[26] J. van Enkhuizen, M. A. Geyer, and J. W. Young. Differential effects of dopamine transporter inhibitors in the rodent iowa gambling task. Psychopharmacology, 225(3):661-674, 2013.

[27] H. Wickham. A layered grammar of graphics. Journal of Computational and Graphical Statistics, 19(1):3-28, 2010.

[28] H. Wickham. ggplot2. Wiley Interdisciplinary Reviews: Computational Statistics, 3(2):180-185, 2011.

[29] L. Wilkinson. The grammar of graphics. In Handbook of computational statistics, pages 375-414. Springer, 2012.

[30] F. D. Zeeb, T. W. Robbins, and C. A. Winstanley. Serotonergic and dopaminergic modulation of gambling behavior as assessed using a novel rat gambling task. Neuropsychopharmacology, 34(10):2329_ 2343, 2009. 